\title{
Bronchiale Provokationsteste
}

\author{
Bronchial Challenge Tests
}

Autor

Institut

\section{Baur}

Ordinariat für Arbeitsmedizin, Universitätsklinikum Hamburg-Eppendorf

Zentralinstitut für Arbeitsmedizin und Maritime Medizin Hamburg (ZfAM) eingereicht 21.9. 2010

akzeptiert nach Revision 11. 10.2010

\section{Bibliografie}

Dol http://dx.doi.org/ 10.1055/s-0030-1255967

Online-Publikation: 10. 12. 2010

Pneumologie 2011; 65:

340-346 ๔ Georg Thieme

Verlag KG Stuttgart · New York ISSN 0934-8387

\section{Korrespondenzadresse}

Prof. Dr. med. Xaver Baur

Ordinariat für Arbeitsmedizin,

Universitätsklinikum

Hamburg-Eppendorf

Zentralinstitut für Arbeits-

medizin

Seewartenstr. 10

20459 Hamburg

Xaver.baur@bsg.hamburg.de

\section{Zusammenfassung \\ $\nabla$}

Es wird eine aktuelle Übersicht über Indikationen, Kontraindikationen, Durchführung und Interpretation bronchialer Provokationsteste gegeben. Das diagnostische Stufenschema des Asthma bronchiale umfasst neben der klinischen Anamnese als Besonderheit die detaillierte Expositions-(Arbeits-)Anamnese, die Lungenfunktionsprüfung, den unspezifischen bronchialen Provokationstest, die allergologische Diagnostik (HautPricktest, Bestimmung spezifischer IgE-Antikörper), Karenz- und Reexpositionstest und als Goldstandard schließlich spezifische bronchiale Provokationsteste. Letzteren kommt im Rahmen der Diagnosesicherung, in Bezug auf gezielte Therapie- und Präventionsmaßnahmen sowie versicherungsrechtliche Aspekte (Frage: Berufskrankheit?) ein hoher Stellenwert zu. Spezifische bronchiale Provokationsteste und deren Variante, der arbeitsplatzbezogene Inhalationstest (AIT), dienen der Objektivierung und dem Ausschluss der klinischen Relevanz und Aktualität einer respiratorischen Sensibilisierung. Sie setzen eine eingehende Erfahrung des Untersuchers voraus. In der ganz überwiegenden Mehrzahl der Erkrankungsfälle sind sie nicht erforderlich, insbesondere nicht, wenn Anamnese, Lungenfunktionsprüfung, Allergieteste, Karenz- und Reexpositionsversuch übereinstimmend positive Resultate liefern. Stehen allergische Symptome der Augenbindehäute oder des oberen Respirationstrakts im Vordergrund, empfiehlt sich die Durchführung eines spezifischen konjunktivalen bzw. nasalen Provokationstestes.

\section{Einleitung}

$\nabla$

In diesem Review wird auf Basis der 1997 bzw. 2002 von Arbeitsgruppen der DGP erstellten Empfehlungen [1,2], neueren Veröffentlichungen und eigener Erfahrungen eine Übersicht über den

\section{Abstract \\ $\nabla$}

This is an updated overview of indications, contraindications, performance and interpretation of bronchial challenge tests. As speciality, the diagnostic step by step scheme comprises in addition to the clinical case history a detailed exposure (occupational) history, lung function testing, assessment of nonspecific bronchial hyperresponsiveness, allergological diagnostics (skin prick test, measurement of specific IgE antibodies), privation and reexposure test and as gold standard specific bronchial challenge tests. The last mentioned tests are of particular importance in the framework of a diagnostic backup with regard to specific therapeutic and preventive measures and insurance regulations (occupational disease?). Specific bronchial challenge tests and their variant, the workplace-related challenge test, serve to objectify or exclude the clinical relevance and the current state of a respiratory sensitization. They require a comprehensive experience of the physician performing the tests. The majority of diseases does not necessitate these tests, especially if case history, lung function testing, allergy tests, privation and reexposure test provide unanimously positive results. If allergic symptoms of conjunctiva or the upper respiratory tract are of prime importance the performance of a specific conjunctival or nasal challenge test is recommended. 


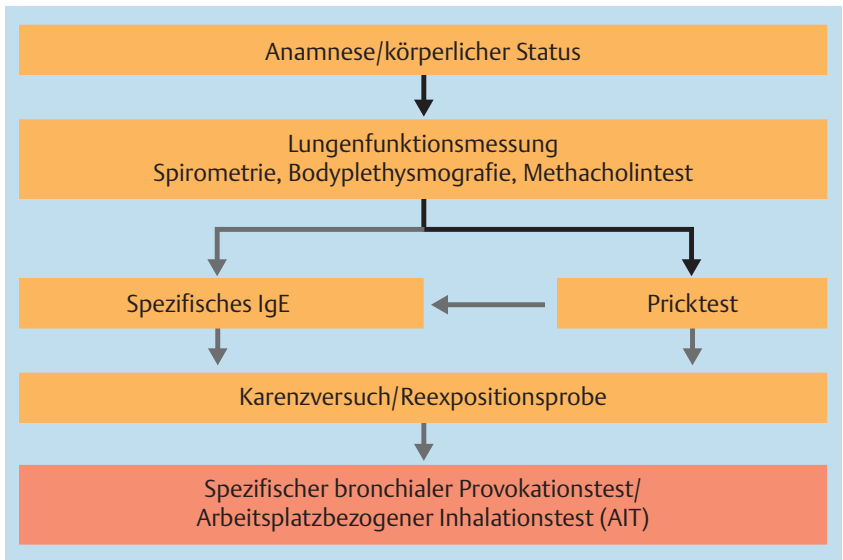

Abb. 1 Diagnostisches Vorgehen bei Verdacht auf Asthma bronchiale. Dunkle Pfeile obligat, helle gestreifte Pfeile fakultativ.

zugt Parasympathomimetika oder Histamin) oder mit spezifischen asthmaauslösenden Noxen (v.a. Allergene) durchgeführt werden. Beide Verfahren, ersteres meist als Screening oder Voruntersuchung, dienen der Diagnostik des Asthma bronchiale im Rahmen eines Stufenschemas ( Abb. 1). Dabei ist zu beachten, dass die unspezifische bronchiale Hyperreaktivität kein für das Asthma bronchiale spezifischer Befund ist; sie kommt auch gehäuft unter Patienten mit allergischer Rhinitis, COPD, exogen-allergischer Alveolitis, Lungenfibrosen und anderen Lungenkrankheiten vor. Andererseits weist sie eine relativ hohe Sensitivität in Bezug auf das Asthma bronchiale auf [3]. Demgegenüber ist der spezifische bronchiale Provokationstest sowohl durch eine hohe Sensitivität als auch Spezifität gekennzeichnet, vorausgesetzt, die Exposition wird mit den im Alltag einwirkenden Substanzen in der üblicherweise vorkommenden Konzentration und Dosis durchgeführt.

\section{Allgemeine Aspekte bronchialer Provokationsteste $\nabla$}

Zunächst ist im Rahmen des individuellen diagnostischen Prozedere $(\bullet$ Abb. 1) $[4,5]$ zu prüfen, ob eine Indikation für bronchiale Provokationsteste gegeben ist. Dies ist v. a. bei folgenden Konstellationen der Fall: unsichere Diagnose, Widerspruch zwischen asthmatischen Beschwerden und normaler Basis-Lungenfunktion, aus dem Ergebnis des Provokationstestes zu erwartende therapeutische und/oder präventive Konsequenzen. Kontraindikationen sind auszuschließen. Diese umfassen relevante Einschränkungen der Lungenfunktion, einen aktuellen Infekt, schwerwiegende Erkrankungen, fehlende Einrichtung zur Notversorgung, eine Therapie mit Bronchospasmolytika und in Bezug auf die spezifische bronchiale Provokation auch die Therapie mit Betablockern. Eine relative Kontraindikation stellt die Schwangerschaft dar. Spezifische Provokationsteste verbieten sich bei in der Vergangenheit aufgetretenen sehr schweren Allergenreaktionen wie Status asthmaticus, Larynxödem, Schock, wenn anhand des Krankheitsverlaufs weiterhin eine entsprechende Reaktionslage anzunehmen ist ( $\bullet$ Tab. 1).

Wichtig ist die Einhaltung genügend langer Pausen von atemwegswirksamen Medikamenten ( Tab. 2).

Der Verlauf der Lungenfunktion wird mittels Ganzkörperplethysmografie oder Spirometrie nach internationalen Qualitätsstandards durchgeführt [6-8]. Als messtechnische Positivgrenze für einen bronchialen Provokationstest (bronchialobstruktive Reak-
Tab. 1 Kontraindikationen für bronchiale Provokationsteste.

1. Relevante Einschränkungen der Lungenfunktion

(Orientierung: $\mathrm{FEV}_{1}<70 \%, \mathrm{sR}_{\mathrm{t}}>1,2 \mathrm{kPa} \times \mathrm{s}$ )

2. Akuter Infekt

3. Schwerwiegende Krankheiten

4. Sehr hohes Risiko, z. B. Status asthmaticus, Larynxödem oder Schockreaktion nach früherer Allergeneinwirkung und anzunehmender persistierender entsprechender Reaktionslage (gilt für spezifische bronchiale Provokationsteste)

5. Therapie mit Bronchodilatatoren

6. Fehlende Einrichtung oder Möglichkeit zur Notfallversorgung

7. Therapie mit Betablockern (relative Kontraindikation bei unspezifischem bronchialen Provokationstest)

8. Schwangerschaft (relative Kontraindikation)

\section{Tab. 2 Medikamentenpause vor bronchialen Provokationtesten.}

\begin{tabular}{|c|c|}
\hline$\beta_{2}$-Sympathomimetika inhalativ (lang wirkende) & $8(24) h$ \\
\hline$\beta_{2}$-Sympathomimetika oral ret. (z. B. Bambec) ${ }^{\circledR}$ & $24 \mathrm{~h}$ \\
\hline Parasympatholytika inhal. (z. B. Atrovent ${ }^{\circledR}$ ) & $12 \mathrm{~h}$ \\
\hline z. B. Spiriva ${ }^{\circledR}$ & $48 \mathrm{~h}$ \\
\hline Theophyllin retardiert & $24 \mathrm{~h}$ \\
\hline Kortikosteroide oral (> 8 mg Prednisonäquivalent) & 2 Wo. \\
\hline Kortikosteroide inhalativ & $12 \mathrm{~h}$ \\
\hline Antihistaminika & $48 \mathrm{~h}$ \\
\hline Cromoglicinsäure (z. B. Intal $\left.{ }^{\circledR}\right)$ & $48 \mathrm{~h}$ \\
\hline$\beta$-Rezeptorenblocker & $12(24) \mathrm{h}$ \\
\hline Leukotrienantagonisten & 5 Tage \\
\hline $\begin{array}{l}\text { Zentral wirkende Antihypertensiva und trizyklische } \\
\text { Psychopharmaka }\end{array}$ & 21 Tage \\
\hline
\end{tabular}

Tab. 3 Positiv-Grenzwerte für bronchiale Provokationsteste.

$$
\begin{aligned}
& \triangle \mathrm{FEV}_{1}:-20 \% ;\left(P D_{20}\right) \\
& \Delta \mathrm{sR}_{\mathrm{aw}}:+65 \%, \text { auf } \geq 2,0 \mathrm{kPa}{ }^{*} \mathrm{~s} ;\left(P D_{65 ; 2 \mathrm{KPa}^{*} \mathrm{~s}}\right) \\
& \Delta \mathrm{sG}_{\mathrm{aw}}:-40 \%, \text { auf } \leq 0,5 / \mathrm{kPa}^{*} \mathrm{~s} ;\left(P D_{40 ; 0,5 / k P a}{ }^{* s}\right)
\end{aligned}
$$

tion) wird ein Anstieg der spezifischen Resistance $\mathrm{sR}_{\mathrm{aw}} \mathrm{um}$ 65\% auf mindestens $2 \mathrm{kPa} \times \mathrm{s}$ (bzw. ein $\mathrm{sG}_{\mathrm{aw}}$-Abfall von $40 \%$ auf 0,5 / $\mathrm{kPa} \times \mathrm{s})^{*}$ gefordert, alternativ ein Abfall der $\mathrm{FEV}_{1}$ um mindestens 20\% ( Tab. 3).

Eine unspezifische bronchiale Hyperreaktivität liegt vor, wenn diese Grenzwerte bei einer am Mund gemessenen Methacholindosis von max. 0,3 mg erreicht werden $\left(\mathrm{PD}_{65 ; 2} \mathrm{kPa}^{*} \mathrm{~s}\right.$ bz. $\left.\mathrm{PD}_{20}\right)$. Die hier zugrunde gelegte Methacholindosis wurde am Vernebler Provocation Test II (Pari) ermittelt [9]; gerätebedingt können sich hiervon Abweichungen ergeben $[10,11]$. Die vernebelte Menge ist etwa doppelt so groß wie die am Mund gemessene.

\section{Unspezifische bronchiale Provokationsteste \\ $\nabla$}

Die Hauptindikationen sind asthmatische Beschwerden in Verbindung mit normalen oder grenzwertigen Lungenfunktionswerten unter Ruhebedingungen. Daneben eignet sich die Methode für Verlaufsbeobachtungen unter bestimmten Risikokonstellationen sowie zur Abschätzung eines möglichen Risikos unter gefährdenden inhalativen Belastungen ( $\bullet$ Tab. 4).

\footnotetext{
Abweichend von der Festlegung in der bisherigen deutschen Provokationstest-Empfehlung [1] entsprechen nach Vergleichsuntersuchungen dem 20\%igen $F_{1} V_{1}$-Abfall sGaw- bzw. sRaw- (Raw-) Änderung von - 40\% (35$50 \%)$ bzw. etwa $+65 \%[13-15]$.
} 
Tab.4 Indikationen für den unspezifischen bronchialen Provokationstest. - asthmatisches Beschwerdebild ohne Nachweis einer Bronchialobstruktion

- Überwachung von Risikoprobanden, z. B. bei erheblicher inhalativer Gefährdung am Arbeitsplatz

- Verlaufsbeobachtung nach spezifischem bronchialen Provokationstest

Tab. 5 Vorgehen beim unspezifischen bronchialen Provokationstest.

- Aufklärung und Einwilligung des Patienten
- Medikamentenpause und Begleittherapie erfragen
- mindestens 4 Konzentrationsstufen bis zum Erreichen der Maximal-
dosis
-5 (6) Stufen zur Vermeidung von Zwischenfällen bei anzunehmender
starker bronchialer Hyperreaktivität (überprüfen, ob es diesbezüglich
anamnestische Hinweise gibt)
- Steigerung der Substanzmenge in Verdopplungsschritten

Die Tab. 5 und 6 fassen das diagnostische Vorgehen zusammen. Nach der Basisuntersuchung der Lungenfunktion wird in ansteigenden Dosen Methacholin (oder eine entsprechende Testsubstanz) inhalativ appliziert [1], wobei nach jedem Schritt eine Kontrolle der Lungenfunktion erfolgt. Neben der traditionellen Durchführung mit dem Provocation Test II der Fa. Pari $[1,12]$ kommen auch übliche Vernebler zum Einsatz [10,11]. Zur Beurteilung wird eine Graduierung des Messergebnisses in Abhängigkeit von der Dosis, bei der das o.g. Positivkriterium erreicht wurde, empfohlen ( $\bullet$ Tab. 7; $\bullet$ Abb. 2).

\section{Spezifische bronchiale Provokationsteste \\ $\nabla$}

Indikationen für den spezifischen bronchialen Provokationstest ergeben sich bei Vorliegen von Diskrepanzen oder unsicheren Aussagen in Anamnese (v.a. hinsichtlich ursächlicher Allergene), Lungenfunktionsprüfung, Hauttestung und IgE-Antikörperbestimmung. Dies gilt auch für den Verdacht auf isolierte verzögerte bronchialobstruktive Reaktionen, die in der Regel anamnestisch nicht sicher zugeordnet werden können, ferner für inhalativ ausgelöste nicht respiratorische Reaktionen, Sensibilisierungen auf neue oder bisher unbekannte Allergene sowie für spezielle
Fragestellungen im Rahmen gutachterlicher Zusammenhangsbeurteilungen. Da der spezifische inhalative Provokationstest mit dem Risiko einer überschießenden allergischen Reaktion einhergeht, ist die Indikation restriktiv zu stellen, d.h. nur dann, wenn sein Ergebnis wesentlich ist für Therapie-Entscheidungen (z.B. Hyposensibilisierung), Prävention (Wohnungs-, Arbeitsplatzwechsel, Maßnahmen nach §3 der Anlage der Berufskrankheitenverordnung) oder Entschädigung (Berufskrankheit).

Die $\odot$ Tab. 8-10 fassen die erforderliche Vordiagnostik, allgemeine Aspekte und spezielle Indikationen zusammen.

\section{Durchführung im Einzelnen}

$\nabla$

Die Indikation für die Provokationstestung wird zunächst nochmals überprüft und das Vorliegen von Kontraindikationen ausgeschlossen. Antiallergische, antiinflammatorische und den Bronchialmuskeltonus beeinflussende Pharmaka müssen entsprechend ihrer Wirkdauer vor der Untersuchung abgesetzt worden sein. Die technische Ausrüstung (übliche Notfallmedikation, Sauerstoff, inhalier- und injizierbare Bronchospasmolytika, Antiallergika, Kortikosteroide, Adrenalin) und geschultes Personal (inklusive eines sachkundigen und in Erste-Hilfe-Maßnahmen geschulten Arztes) müssen für Notfälle (Status asthmaticus-Therapie, Schockbehandlung, kardiopulmonale Reanimation) vorhanden sein.

Der Patient ist über die beabsichtigte Untersuchung einschließlich deren Risiken aufzuklären; seine schriftliche Einwilligung ist einzuholen.

Die Ergebnisse der Basisuntersuchung (körperlicher Status, allergologische Diagnostik, Lungenfunktionsprüfung, unspezifischer bronchialer Provokationstest) sind vom Arzt zu überprüfen und hinsichtlich der festzulegenden Anfangs- und Gesamtdosis sowie der einzelnen Dosissteigerungsschritte bzgl. der Testsubstanz zu berücksichtigen (s.u.).

Am weitesten verbreitet und in der Anwendung am einfachsten ist die Applikation der Kontrolle (Placebo) und der Testsubstanz über einen Vernebler. Dies setzt voraus, dass wässrige Lösungen vorliegen, die standardisiert sein sollen. Eine Alternative ist die Exposition gegenüber gasförmigen Stoffen, z.B. Isocyanaten, in einer Expositionskammer. Dabei sollte die Luftkonzentration fortlaufend überprüft werden. Dies kann auch mit dem sog.

\begin{tabular}{|c|c|c|c|c|c|}
\hline $\begin{array}{l}\text { Die folgenden Punkte beziehen si } \\
\text { (Beutel) geatmet wird und die Me } \\
\text { kann sich vor allem infolge eines } \\
\text { abweichende Berechnungsgrund }\end{array}$ & $\begin{array}{l}\text { auf den } \mathrm{Pr} \\
\text { ng der ve } \\
\text { eren Tröp } \\
\text { e evtl. auc }\end{array}$ & $\begin{array}{l}\text { on Test } \\
\text { iten MC } \\
\text { ektrum } \\
\text { deres V }\end{array}$ & $\begin{array}{l}\text { vobei da } \\
\text { perücks } \\
\text { ergeber }\end{array}$ & $\begin{array}{l}\text { rosol } \\
12] .(1 \\
\text { if Aer }\end{array}$ & $\begin{array}{l}\text { Reservoir } \\
\text { ר Geräten } \\
\text { schläge eine }\end{array}$ \\
\hline Die eingesetzte MCH-Konzentrati & beträgt 3 , & L (z. B. I & & & \\
\hline Vernebler-Laufzeit (s) & $6^{*}$ & 12 & 24 & 48 & 96 \\
\hline MCH-Einzeldosis im Beutel (mg) & $0,015^{*}$ & 0,030 & 0,061 & 0,122 & 0,243 \\
\hline kumulative MCH-Dosis (mg) & $0,015^{*}$ & 0,046 & 0,106 & 0,228 & 0,471 \\
\hline
\end{tabular}

${ }^{*}$ Kann entfallen, falls eine stärkere bronchiale Hyperreaktivität unwahrscheinlich ist.
Tab. 6 Dosierungsschema des unspezifischen Provokationstestes mit nur einer Methacholin (MCH)-Konzentration.

Tab. 7 Graduierung der unspezifischen bronchialen Hyperreaktivität. 

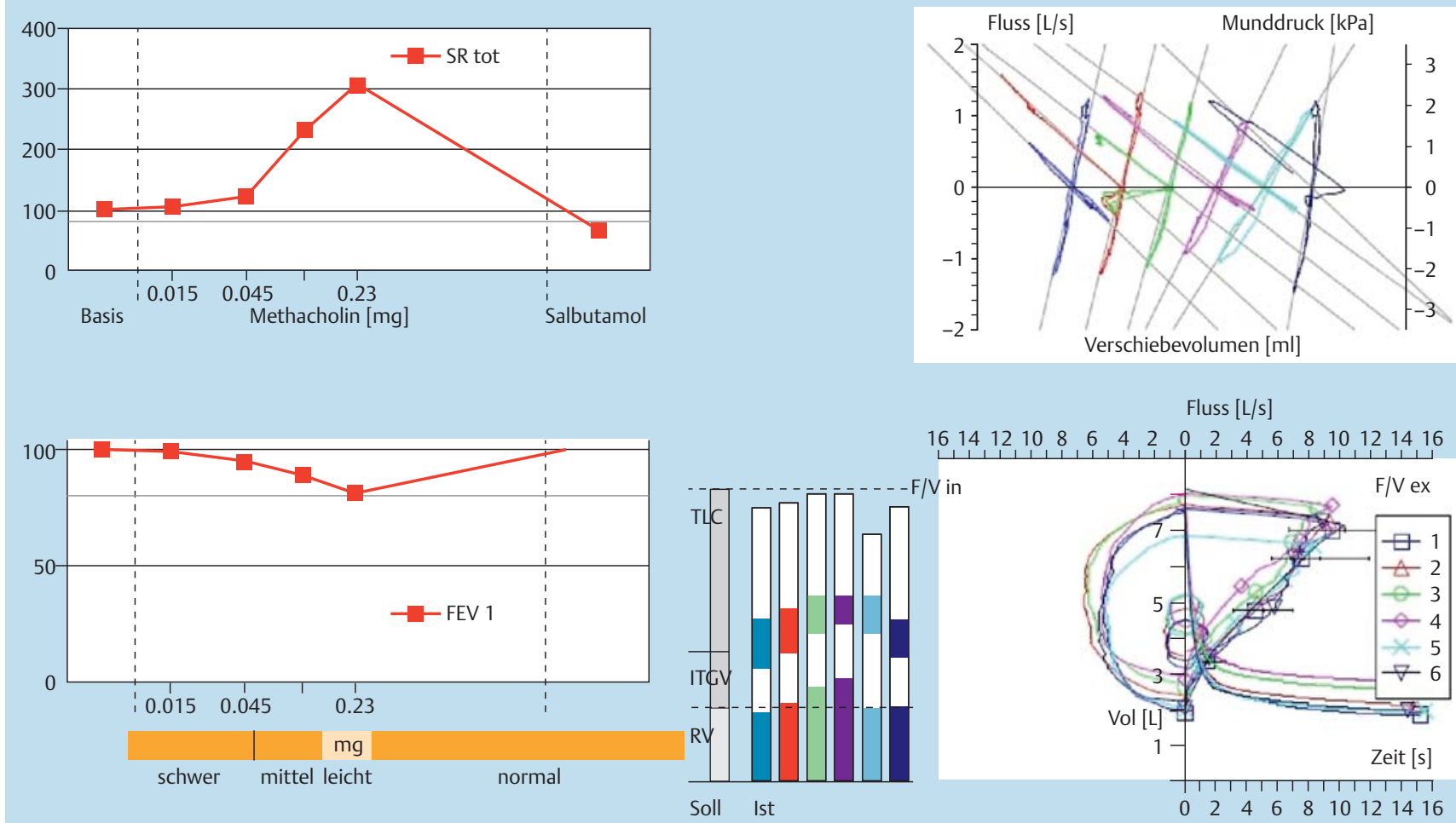

Abb. 2 Beispiel eines unspezifischen bronchialen Provokationstestes mit Methacholin mit Graduierung der Hyperreaktivität (s. auch $\bullet$ Tab. 7).

Closed circuit-System, in dem der Patient definierte Konzentrationen der Testsubstanz aus einem Vorratsgefäß atmet, erfolgen. Hiervon zu unterscheiden sind Expositionsteste (AIT) mit staubförmigen Aerosolen, z. B. Mehlstaub, die häufig auch im Rahmen eines arbeitsplatzbezogenen inhalativen Expositionstestes (AIT) angewandt werden.

Initial erfolgt eine Placebotestung mit einer geeigneten Kontrollsubstanz (Allergen-Lösungsmittel). Diese Placebotestung wird in der Regel als Kurzzeittest durchgeführt, es sei denn, dass von einer erheblich variierenden Bronchialobstruktion auszugehen ist; in einem solchen Fall sollte vorab zusätzlich ein Tagesprofil der Lungenfunktion dokumentiert werden.

Die inhalative Applikation der Testsubstanz soll in einer geschlossenen, zwangsentlüfteten Kabine mit Ausatemfilter stattfinden. Die Anfangsdosis muss unter Berücksichtigung der üblichen, Beschwerden auslösenden Belastungen in der Umgebung, der bronchialen Reaktivität und des Sensibilisierungsgrades (s. Hauttest- und spezifischen IgE-Befund) so niedrig gewählt werden, dass noch keine Reaktion zu erwarten ist (Orientierung: Pricktest-Schwellenkonzentration).

Die Dosis wird bei Ausbleiben einer Reaktion nach 10 bis 15 min um einen Faktor von etwa 3 (2-4) gesteigert. Wenn eine Lungenfunktionsänderung bereits nahe am Positivkriterium festzustellen ist, wird die zuletzt applizierte Dosis nochmals verabreicht. Die Lungenfunktionsprüfung (Spirometrie oder Bodyplethysmografie) erfolgt zu Untersuchungsbeginn, nach Placeboapplikation und nach jeder Dosis der Testsubstanz; empfohlen werden darüber hinaus im Anschluss daran Messungen nach $15 \mathrm{~min}$, 30 min, 45 min, 1 Std., 2 Std., 4 Std., 6 Std., zusätzlich, wenn Atemnot auftritt und wenn Hinweise auf eine verzögerte Reaktion bestehen. Der Patient muss bis zur 6. Stunde, ggf. länger, bis zur Normalisierung der Werte und Beschwerdefreiheit unter Beobachtung bleiben. Ergänzend zu den vorgenannten Messungen nach der Provokation sollte stündlich bis zur 5. Stunde eine Ei-
Tab. 8 Vorab-Diagnostik bei bronchialen Provokationstesten (ist auch zur Risikoabschätzung erforderlich).

- Anamnese (Larynxödem, schwere Asthma- oder systemische
Reaktionen im relevanten Zeitraum?)
- körperlicher Status
- Lungenfunktion (Spirometrie, Flussvolumenkurve, Bodyplethysmo-
grafie)
- Methacholintest (Grad der BHR?)
- Allergie-Hauttest (Pricktest) oder spezifisches IgE (Sensibilisierungs-
grad?)
- Messung oder wenigstens qualitative und quantitative Abschätzung
der als krankheitsursächlich vermuteten inhalativen Belastung zu
Hause, in der allgemeinen Umwelt, am Arbeitsplatz etc.

Tab.9 Allgemeine Aspekte der Indikationsstellung für den spezifischen bronchialen Provokationstest

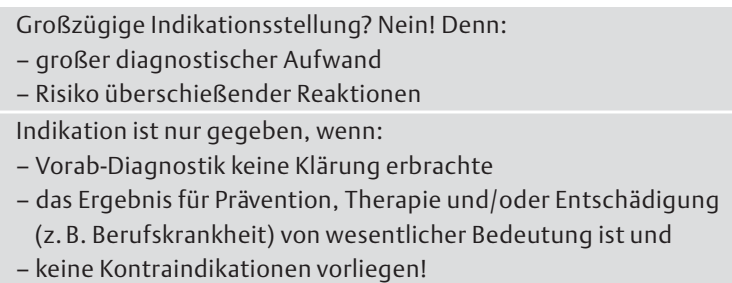

genmessung des Patienten mittels eines portablen Spirometers erfolgen, in komplizierten Fällen länger. Besteht der Verdacht auf eine exogen-allergische Alveolitis, sind initial und mindestens 4 und 6 Std. nach Provokation $\mathrm{D}_{\mathrm{L}, \mathrm{CO}}$, Blutgase, Körpertemperatur und Leukozyten im peripheren Blut zu bestimmen.

Der Patient muss nach der Applikation der Testsubstanz ein inhalierbares Beta-2-Sympathomimetikum bei sich haben und instruiert sein, wie er sich bei Auftreten von Atembeschwerden zu verhalten hat und den Arzt erreicht. 
1. Unsichere Diagnose (Diskrepanzen oder unsichere Aussagen in Anamnese, Hauttest, spezifischer IgE-Bestimmung) vor Einleitung weitreichender Präventions- und Therapiemaßnahmen wie Wohnungs-, Arbeitsplatzwechsel oder Hyposensibilisierung. Beachte: Das Fehlen einer unspezifischen bronchialen Hyperreaktivität schließt ein Asthma bronchiale nicht aus; deshalb sollten bei entsprechend starken Hinweisen die weiteren diagnostischen Schritte (s. Abb. 1) erfolgen

2. Gutachterliche Stellungnahme (Diagnosesicherung; differenzialdiagnostische Abklärung)

3. Verdacht auf bronchiale Sensibilisierung gegen neue oder bisher unbekannte Allergene

4. Verdacht auf isolierte verzögerte Bronchialobstruktion

5. Fragliche asthmatische Reaktion auf p. o. oder i.v. zugeführte Substanzen

6. Verdacht auf inhalativ ausgelöste nicht-respiratorische Reaktionen
Tab. 10 Indikationen für den spezifischen bronchialen Provokationstest im Einzelnen.

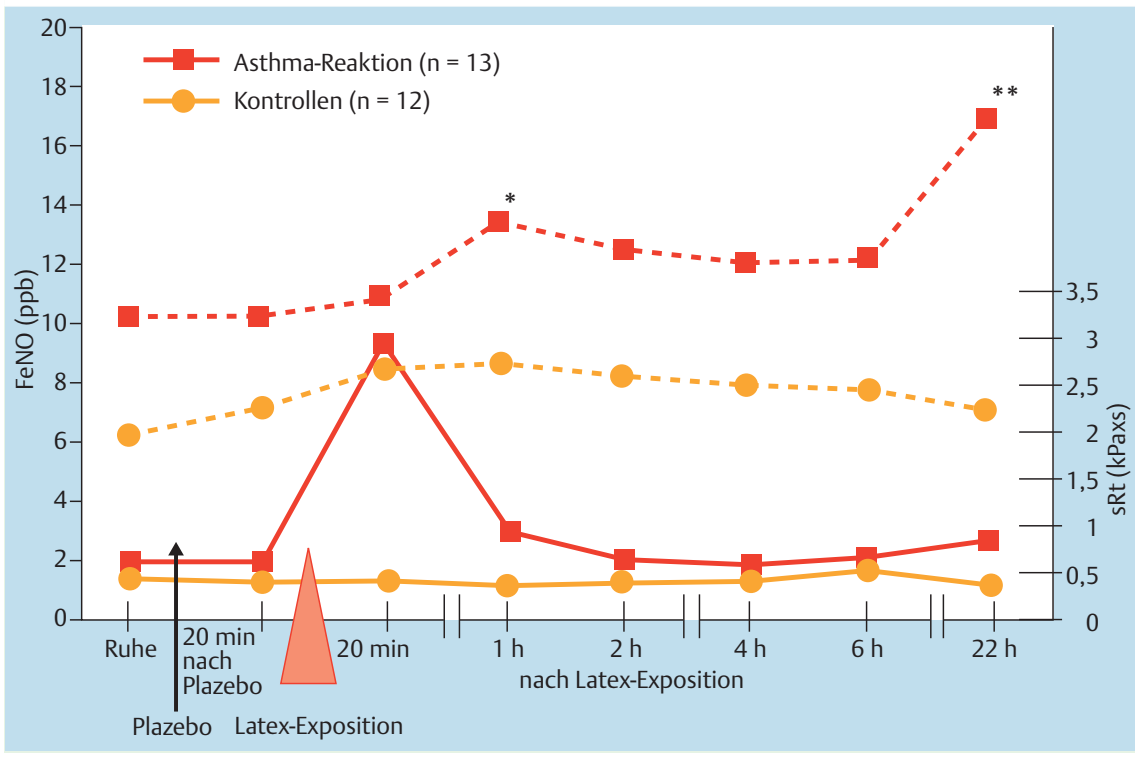

Abb. 3 Bronchialobstruktive Sofortreaktionen und FeNO-Veränderungen in bronchialen Provokationstesten mit gepuderten Latexhandschuhen. $\mathrm{FeNO}=$ exhalierte NO-Fraktion; $\mathrm{sR}_{\mathrm{t}}=$ spezifischer Atemwegswiderstand. Angegeben sind die Mittelwerte der 13 Asthmatiker und der 12 Kontrollen. ${ }^{*} p<0,05 ;{ }^{*} p<0,01$. Durchgezogene Linien: $s R_{t}$; gestrichelte Linien: FeNO.

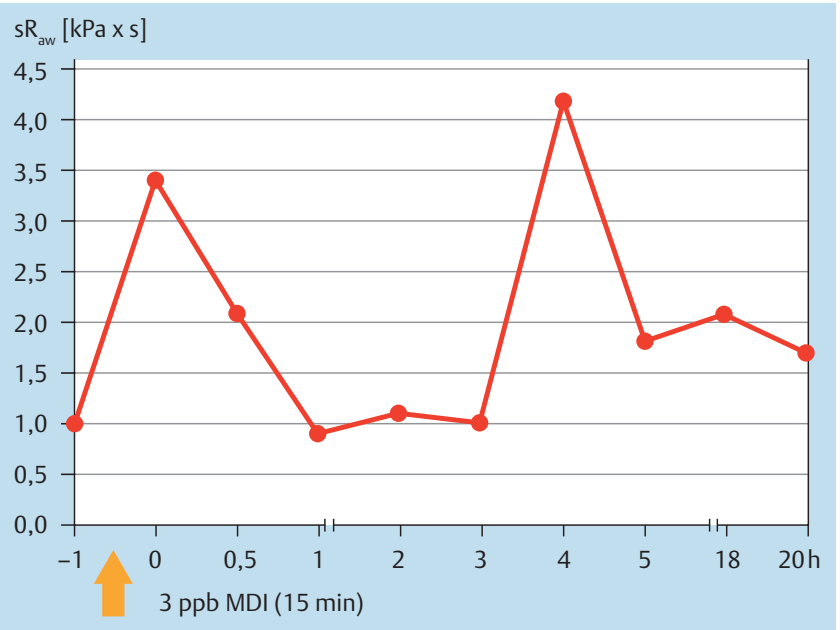

Abb. 4 Arbeitsplatzbezogener inhalativer Expositionstest mit dem Isocyanat Diphenylmethan-4,4'-Diisocyanat (MDI). Der Isocyanatarbeiter entwickelt eine duale asthmatische Reaktion. Am Kontrolltag ist die Lungenfunktion durchweg normal ( $\mathrm{ppb}=$ parts per billion [Milliarde]; $\mathrm{s}_{\mathrm{aw}}=$ spezifischer Atemwegswiderstand [in $\mathrm{kPa} \times \mathrm{s}$ ], ganzkörperplethysmografisch bestimmt).

An einem Untersuchungstag soll wegen der Möglichkeit einer verzögerten Reaktion nur eine Substanz getestet werden.

Eine Besonderheit stellt der arbeitsplatzbezogene inhalative Expositionstest dar, z. B. mit gepuderten Latexhandschuhen (s. Beispiele in $\bigcirc$ Abb. 3), Mehl- oder Getreidestaub oder gasförmigen Isocyanaten [16,17] http://www.dgaum.de/index.php/publikationen/leitlinien/leitlinienarbeitsmedizin/268-llait?format=pdf. Bzgl. Besonderheiten im Rahmen der Testung von speziellen Ar-
Tab. 11 Besonderheiten und diagnostische Limitierungen des spezifischen bronchialen Provokationstestes und AIT.

- In einem Teil der Fälle treten erst nach wiederholten spezifischen
Provokationen Steigerungen von BHR und/oder bronchialobstruktive
Reaktionen auf.
- Das Spirometrie-(PEF)-Monitoring während der üblichen Exposition
(z. B. Arbeitsschichten) und ohne dieselbe ist wegen der dabei reellen
Bedingungen dem spezifischen Provokationstest vorzuziehen.
Ansonsten sollte bei gegebener Indikation die spezifische Provoka-
tionstestung (bzw. AIT) erfolgen, falls die entsprechende Fachkom-
petenz vor Ort vorhanden ist.
- Bei Fortbestehen von Asthmasymptomen sind bei negativen Test-
ergebnissen die Untersuchungen nach mehreren Monaten zu wieder-
holen, da die Möglichkeit des noch nicht fassbaren Vorstadiums eines -
Asthmas besteht.

beitsstoffen wird auf die weiterführende Literatur verwiesen $[8,18-21]$.

\section{Abbruchkriterien und Beurteilung spezifischer bronchialer Provokationsteste}

Der Provokationstest wird abgebrochen bei Auftreten von erheblicher Atemnot in Verbindung mit einem pathologischen Auskultationsbefund, anderen wesentlichen Beschwerden oder Erreichen eines Positivkriteriums (Anstieg des spezifischen Atemwegswiderstands, $\mathrm{sR}_{\mathrm{aw}}$, um mindestens $65 \%$ auf $\geq 2 \mathrm{kPa}^{*} \mathrm{~s}$ oder Abfall der $\mathrm{FEV}_{1}$ um $\left.\geq 20 \%\right)(\bullet$ Tab. 3).

Symptome wie Husten, Atemnot, Giemen und Brummen, Fließschnupfen, Niesattacken u. dgl. m. sind zu dokumentieren. 


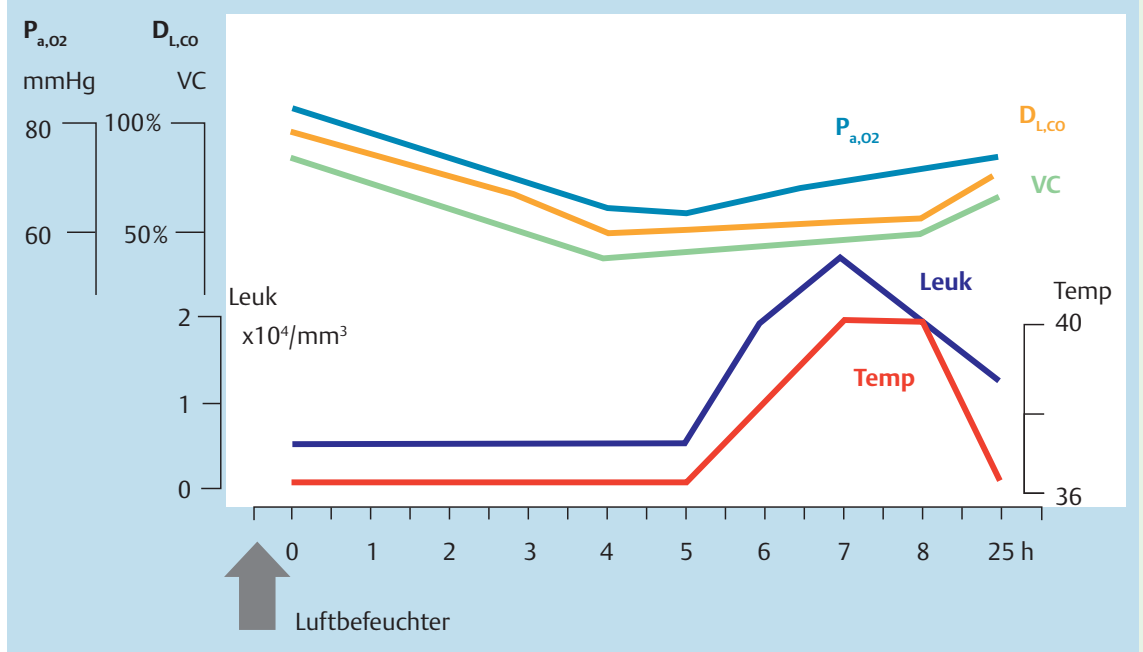

Abb. 5 Akute exogen-allergische Alveolitis (Beleuchterlunge) mit restriktiver Ventilationsstörung, Gasaustauschstörung und systemischer Reaktion nach 30-minütiger Exposition gegenüber Luftbefeuchteraerosolen.

$\mathrm{P}_{\mathrm{a}, 02}=$ Sauerstoffpartialdruck

$\mathrm{D}_{\mathrm{L}, \mathrm{CO}}=\mathrm{CO}-$ Diffusionskapazität (\% Soll)

VC = Vitalkapazität (\% Soll)

Leuk $=$ Leukozyten im peripheren Blut

Temp $=$ Körpertemperatur $\left({ }^{\circ} \mathrm{C}\right)$

Der Anstieg von Stickstoffmonoxid in der exhalierten Luft (FeNO) etwa einen Tag nach Allergenbelastung spricht für eine Typ I-Allergie.

In der weit überwiegenden Mehrzahl der Fälle werden bronchialobstruktive Sofortreaktionen beobachtet ( Abb.3). In etwa $20 \%$ treten duale ( Abb. 4), vereinzelt (vorwiegend durch chemische Noxen) isolierte, verzögerte, rekurrierende oder prolongierte bronchialobstruktive Reaktionen auf. Hiervon zu unterscheiden ist eine nach mehreren Stunden sich entwickelnde restriktive Ventilationsstörung mit systemischen Krankheitszeichen, die eine exogen-allergische Alveolitis anzeigt ( $\bullet$ Abb.5). Neben vorherrschenden linearen werden auch nicht-lineare Dosis-Wirkungs-Beziehungen beobachtet. Vandenplas und Malo [19] sowie Vandenplas et al. [22] wiesen darauf hin, dass in einigen Fällen erst die wiederholte Exposition zu einer signifikanten bronchialobstruktiven Reaktion führt. Hinzuweisen ist zudem auf subklinische Reaktionen, die sich durch eine Steigerung der unspezifischen bronchialen Hyperreaktivität auszeichnen und durch eine Wiederholung des unspezifischen bronchialen Provokationstestes nach 6 bis 24 Stunden erfasst werden können [22]. Dabei sind typischerweise auch Anstiege der inflammatorischen Marker festzustellen.

\section{Besonderheiten sowie diagnostische und technische Grenzen des spezifischen bronchialen Provokations- testes und des arbeitsplatzbezogenen inhalativen Expositionstestes (AIT)}

Die Tab. 11 und 12 weisen auf Besonderheiten und Möglichkeiten falsch positiver und falsch negativer Ergebnisse spezifischer bronchialer Provokationsteste hin.
- Die vermutlich krankheitsauslösende Substanz (z. B. Arbeitsstoff) steht nicht zur Verfügung, kann im Labor nicht appliziert (vernebelt) werden oder es handelt sich um eine stark irritative oder toxisch wirkende Substanz mit erheblichem unspezifischem Reizpotenzial (Test meist ethisch nicht vertretbar und nicht aussagefähig).

- Für die Krankheitsauslösung sind offensichtlich hohe Expositionen (erhebliche Grenzwertüberschreitungen) verantwortlich; aus ethischen Gründen verbietet sich in der Regel eine derartige Provokationstestung.

- Die Wirkung einer lang anhaltenden Exposition lässt sich im spezifischen bronchialen Provokationstest nicht erfassen.

Beachte Sondersituationen am Arbeitsplatz!

Nicht selten erfolgen am Arbeitsplatz Überschreitungen der Arbeitsplatzgrenzwerte, die für die Induktion des vorliegenden Krankheitsbildes verantwortlich gemacht werden können. Unter solchen Bedingungen schließt das negative Ergebnis eines arbeitsplatzbezogenen inhalativen Expositionstests bis zum Arbeitsplatzgrenzwert eine Berufskrankheit nicht aus.

- Der spezifische bronchiale Provokationstest ist bei korrekter Durchführung in der Diagnostik des exogenen Asthma bronchiale der „Goldstandard“.

- Es lassen sich verschiedene Reaktionstypen und Diagnosen erfassen: bronchialobstruktive Reaktionen (sofort auftretende, verzögerte, duale, rekurrierende, prolongierte Reaktion); exogen-allergische Alveolitis (verzögerte restriktive Veränderung mit Gasaustauschstörung und systemischen Entzündungszeichen); subklinische Reaktionen mit Induzierung/Steigerung einer BHR; keine obstruktive und keine restriktive Lungenfunktionsänderung, keine Änderung der bronchialen Reaktivität, keine sonstige Organreaktion und keine systemischen Veränderungen.

- Irritanzien können einen Anstieg der BHR auslösen, dies ist unabhängig vom Reaktionstyp; hinsichtlich der Interpretation ist dabei die reelle Expositionshöhe zu berücksichtigen.

- Erst nach repetetiver spezifischer bronchialer Provokation kommt es gelegentlich zu einer Asthmareaktion (ist v. a. nach längerer Karenz zu beobachten).

- Eine sorgfältige Durchführung des spezifischen bronchialen Provokationstestes vermeidet falsch negative Resultate (z. B. durch ein nicht relevantes Testagens oder eine zu niedrige Dosis).

- Bei Fortbestehen von Asthma-Symptomen sind bei negativen Provokationstestergebnissen die Untersuchungen nach 1-6 Monaten zu wiederholen, da die Möglichkeit des Vorstadiums eines Asthmas besteht.

- Das Ergebnis erlaubt oft die Beantwortung der Fragen: Liegt eine Berufskrankheit vor? Sind Präventionsmaßnahmen (entsprechend §3 Abs.1 der Berufskrankheitenverordnung) erforderlich?
Tab.12 Technisch bedingte Grenzen des spezifischen bronchialen Provokationstestes und des AIT.

Tab. 13 Schlussfolgerungen und wesentliche Aspekte des spezifischen bronchialen Provokationstestes. 
Diese sind durch sorgfältige Planung weitestgehend vermeidbar. In jedem Fall sind sie bei der diagnostischen Beurteilung des Einzelfalles zu berücksichtigen.

Die Testung mit Irritanzien bedarf besonderer Erfahrung. Hier sind unspezifische Effekte (Symptome und Bronchokonstriktion bei unspezifischer Hyperreaktivität) von pathogenetisch kausalen Einwirkungen abzutrennen und nicht selten „falsch-negative“ Testergebnisse unter Berücksichtigung der reellen Expositionsbedingungen auszuschließen.

Detaillierte repetitive Provokationstestungen mit Arbeits- und Umweltstoffen zeigen, dass in einigen Fällen erst die wiederholte inhalative Exposition eine unspezifische bronchiale Hyperreaktivität und/oder eine bronchialobstruktive Reaktion auszulösen vermag. Solche kumulativen Effekte sollten vom Arzt durch engmaschige Verlaufsbeobachtungen verfolgt werden; sie sind entsprechend der reellen Situation (Dauer und Höhe der Exposition) in der Beurteilung des Provokationstestes zu berücksichtigen.

Meist wird der spezifische bronchiale Provokationstest unter Ruhebedingungen durchgeführt, während üblicherweise, z.B. am Arbeitsplatz, mehr oder weniger körperliche Belastungen stattfinden, die mit einer Erhöhung des Atemminutenvolumens und damit der inhalativen Schadstoffaufnahme einhergehen. Um dies zu berücksichtigen, können während der Exposition körperliche Belastungen, z. B. mit 25 Watt auf dem Fahrradergometer, vorgenommen werden.

Wesentliche, meist technisch bedingte Limitationen bronchialer Provokationsteste sind in $\bullet$ Tab. 12 dargestellt.

\section{Schlussfolgerungen}

Wie Tab. 13 zusammenfassend auflistet, erlaubt der spezifische bronchiale Provokationstest wichtige, mit keinem anderen diagnostischen Verfahren erzielbare Aussagen [19,20,23].

Indikationen ergeben sich insbesondere, wenn die erörterten einfacheren Untersuchungen nicht durchführbar sind oder nicht aussagefähige oder widersprüchliche Ergebnisse liefern. Der Provokationstestbefund ist nicht selten für gezielte Präventionsmaßnahmen (z. B. Wohnungssanierung, Arbeitsplatzwechsel) und die Behandlung (Hyposensibilisierung) essenziell. Die sach- und fachgerechte Durchführung lässt das Risiko auf ein Minimum reduzieren. Falsch positiv und falsch negative Testergebnisse sind in der Hand des Erfahrenen weitgehend auszuschließen.

Die Indikation für die unspezifische bronchiale Provokationstestung ist großzügig zu stellen, da es sich um ein relativ einfaches, gut standardisiertes Verfahren handelt, das keine besonderen technischen Anforderungen stellt und einen hohen prädiktiven Wert aufweist. Dagegen sind spezifische bronchiale Provokationsteste aufwendig, sie werden auch nicht entsprechend des Aufwands von den Sozialversicherungen honoriert. Ein gewisses Problem stellt ihre sowohl national als auch international fehlende Standardisierung dar. Eine Arbeitsgruppe der ERS will sich nun dieser Aufgabe annehmen. Es bleibt zu hoffen, dass dieses Review hierzu ebenfalls beiträgt.

\section{Abkürzungen \\ $\nabla$ \\ AIT Arbeitsplatzbezogener inhalativer Expositionstest \\ BHR Unspezifische bronchiale Hyperreaktivität \\ $\mathrm{MCH}$ Methacholin \\ PEF Peak expiratory flow}

\section{Interessenkonflikt}

$\nabla$

Der Autor gibt an, dass kein Interessenkonflikt besteht.

\section{Literatur}

1 Klein G. Empfehlungen zur Durchführung bronchialer Provokationstests mit pharmakologischen Substanzen. Med Klin 1997; 8: 458 - 463

2 Gonsior E, Henzgen M, Jorres RA et al. Guidelines for conducting provocation tests with allergens. German Society of Allergology and Clinical Immunology and German Society for Immunology. Pneumologie 2002; 56: $187-198$

3 Spina D. Bronchial hyperresponsiveness: a rather irritable subject. Clin Exp Allergy 2010; 40: 9-11

4 Wahl $R$, Krause $R$. Methoden der in-vitro-Allergiediagnostik und deren Stellenwert unter Berücksichtigung ihrer technischen Aspekte. Allergologie 2010; 33: 121 - 133

5 Baur X, Preisser A, Oldenburg $M$ et al. Lungenfunktionsprüfungen in der Arbeitsmedizin. Arbeitsmedizinische Leitlinie der Deutschen Gesellschaft für Arbeitsmedizin und Umweltmedizin (DGAUM). Arbeitsmed Sozialmed Umweltmed 2010; 45: 126-134

6 Miller MR, Crapo R, Hankinson J et al. General considerations for lung function testing. Eur Respir J 2005; 26: 153 - 161

7 Miller MR, Hankinson J, Brusasco Vet al. Standardisation of spirometry. Eur Respir J 2005; 26: 319-338

8 Merget R. Bronchiale Provokation. Atemw Lungenkrkh 2010; 36: 189192

9 Mohorn M, Schafer R. Technical aspects in the Pari provocation test I. Pneumologie 1991; 45 Suppl 2: 651 - 653

10 Merget R, Jorres RA, Heinze E et al. Development of a 1-concentration4-step dosimeter protocol for methacholine testing. Respir Med 2009; 103: $607-613$

11 Jorres RA, Nowak D, Kirsten D et al. A short protocol for methacholine provocation testing adapted to the Rosenthal-Chai dosimeter technique. Chest 1997; 111: 866 - 869

12 Ammon J, Marek W, Baur X. Erstellung eines Normalkollektivs für den Metacholin-Provokationstest. Atemw Lungenkrkh 1993; 19: 303 - 304

13 Cockcroft DW, Berscheid BA. Measurement of responsiveness to inhaled histamine: comparison of FEV1 and SGaw. Ann Allergy 1983; 51: $374-$ 377

14 Fish JE, Kelly JF. Measurements of responsiveness in bronchoprovocation testing. J Allergy Clin Immunol 1979; 64: 592-596

15 Frolund L, Madsen F, Svendsen UG et al. Reproducibility of responsiveness to a standardized bronchial allergen provocation-Rt compared to FEV1 as measurement of response to provocation. Clin Allergy 1987; 17: $217-228$

16 Woitowitz HJ, Valentin H, Krieger HG. [Obstructive diseases of the respiratory tract due to chemically irritative or toxic substances (author's transl)]. Prax Klin Pneumol 1979; 33: 1161-1167

17 Baur X, Drexler H, Kraus T et al. Arbeitsmedizinische Leitlinie der Deutschen Gesellschaft für Arbeitsmedizin und Umweltmedizin e.V. (DGAUM). Arbeitsplatzbezogener Inhalationstest (AIT). Arbeitsmed Sozialmed Umweltmed 2010; 45: 434-441

18 Baur X. Crashkurs Lungenfunktion. München-Deisenhofen: Dustri 2009

19 Vandenplas O, Malo JL. Inhalation challenges with agents causing occupational asthma. Eur Respir J 1997; 10: 2612 - 2629

20 Rioux JP, Malo JL, L'Archeveque J et al. Workplace-specific challenges as a contribution to the diagnosis of occupational asthma. Eur Respir J 2008; 32: 997-1003

21 Pepys J, Hutchcroft BJ. Bronchial provocation tests in etiologic diagnosis and analysis of asthma. The American review of respiratory disease 1975; 112: 829-859

22 Vandenplas O, Delwiche JP, Jamart J et al. Increase in non-specific bronchial hyperresponsiveness as an early marker of bronchial response to occupational agents during specific inhalation challenges. Thorax 1996; 51: $472-478$

23 Baur X, Huber H, Degens PO et al. Relation between occupational asthma case history, bronchial methacholine challenge, and specific challenge test in patients with suspected occupational asthma. Am J Ind Med 1998; 33: $114-122$ 